\title{
Simulation Study of CSI Relay over Nakagami-m Fading Channels
}

\author{
JiHua Huang \\ School of information technology, Kunming University, 650214, China \\ email: hjuh1225@163.com
}

Keywords: Nakagami-m; Channel Model; Channel State Information (CSI); composite fading channels; Relay.

\begin{abstract}
Mobile radio channel models are essential for the development, evaluation and test of current mobile radio communication systems and also crucial for the realization of the future systems. Nakagami-m distribution is important for channel modeling in mobile communication. It provides accurate characterization of the simultaneous occurrence of multipath fading and shadowing effects. This paper investigates the performance of linear transceivers in mobile communication systems where channel state information (CSI) is available at the relay station. We simulation study the delays with CSI over Nakagami-m Fading Channels.
\end{abstract}

\section{Introduction}

In this day and age, mobile communication has become an important part of modern life. It is hard to imagine the world without wireless communication. On the other hand, fading as an effect significantly degrades radio signals during wireless propagation. A traditional method to address this phenomenon is using statistical distributions.

There are some classical distributions which are use to model fading channel. For example, Rayleigh, Rice, Lognormal channel model [1]. However, Nakagami-m distribution has gained more attention lately, due to the fact that it provides the best fit for a wider range of the fading amplitudes of mobile channels and fits experimental data more accurately than Gauss, Rayleigh, Rice and log-normal distributions [2]. It is a fading model that accounts for the small-scale variations of the fading signal in a line-of-sight (LOS) communication scenario. Nakagami-m distribution can be a good description of multipath fast fading, with different values of $\mathrm{m}$, which conforms to the Gaussian distribution, Rayleigh distribution and the Rice distribution [3].

In this paper, we study the delays in Nakagami-m [4] fading channel based on mathematically analyses and simulation. The next section presents the theoretical background of Nakagami-m distribution. Section III provides simulation analysis of relays in Nakagami-m fading channel. Finally, the conclusions are drawn in Section IV.

\section{Nakagami-m Fading Distribution}

In wireless communication, the received signal may fluctuate violently and fading gradually due to the multipath propagation. Nakagami-m distribution is able to well characterize the fading signals and provide the accurate fitting ability to both urban and indoor multipath propagation environments. Another important advantage of Nakagami-m distribution is that it can deduce to a large number of multipath channels via adjusting parameter m, which makes it easy to model different kinds of fading propagation.

In the Nakagami-m fading channel model, the p.d.f. of the envelope of the transmitted signal at a single point in time is given by $[3,4]$ :

$$
p(x)=\frac{2 m^{m} x^{2 m-1}}{\Omega^{m} \Gamma(m)} \exp \left(-\frac{m x^{2}}{\Omega}\right), m \geq \frac{1}{2}, x \geq 0
$$

where, $x$ is the received signal level, $\Omega=E\left[x^{2}\right]$ is the average power of the multipath scattering field, and $m$ is the parameter of fading depth, which controls the shape of the distribution and describes the fading degree of the propagation field caused by the interference of the scattering 
and the multipath phenomena. $m$ define as:

$$
m=\Omega^{2} / E\left\{\left[x^{2}-\Omega^{2}\right]^{2}\right\}
$$

In the above formulas, $E[\cdot]$ denotes expectation.

Nakagami-m distribution formula, includes both the Rayleigh distribution and one-sided Gaussian distribution as special cases of $m=1$ and $m=0.5$ respectively. The case $m \rightarrow \infty$ describes the channel without fading. With certain restrictions the Nakagami-m distribution can approximate the Rice distribution [4].

In this paper, we choose to analyze the Nakagami-m channel model due to the fact that other models can be described by the Nakagami-m distribution by an appropriate choice of relevant parameters.

\section{Simulation Analysis of Relays in Nakagami-m fading channel}

The classification of relays is shown in Fig. 1.

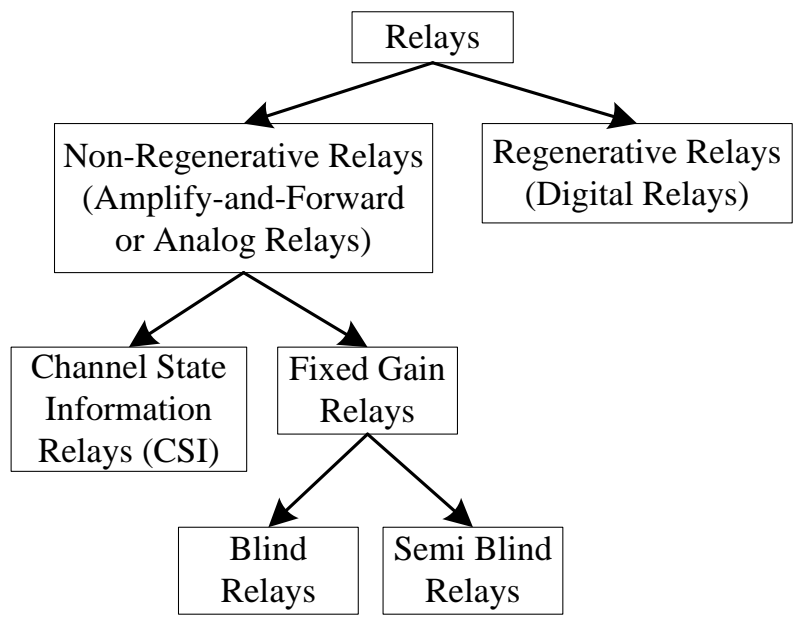

Fig. 1 Classification of Raleys
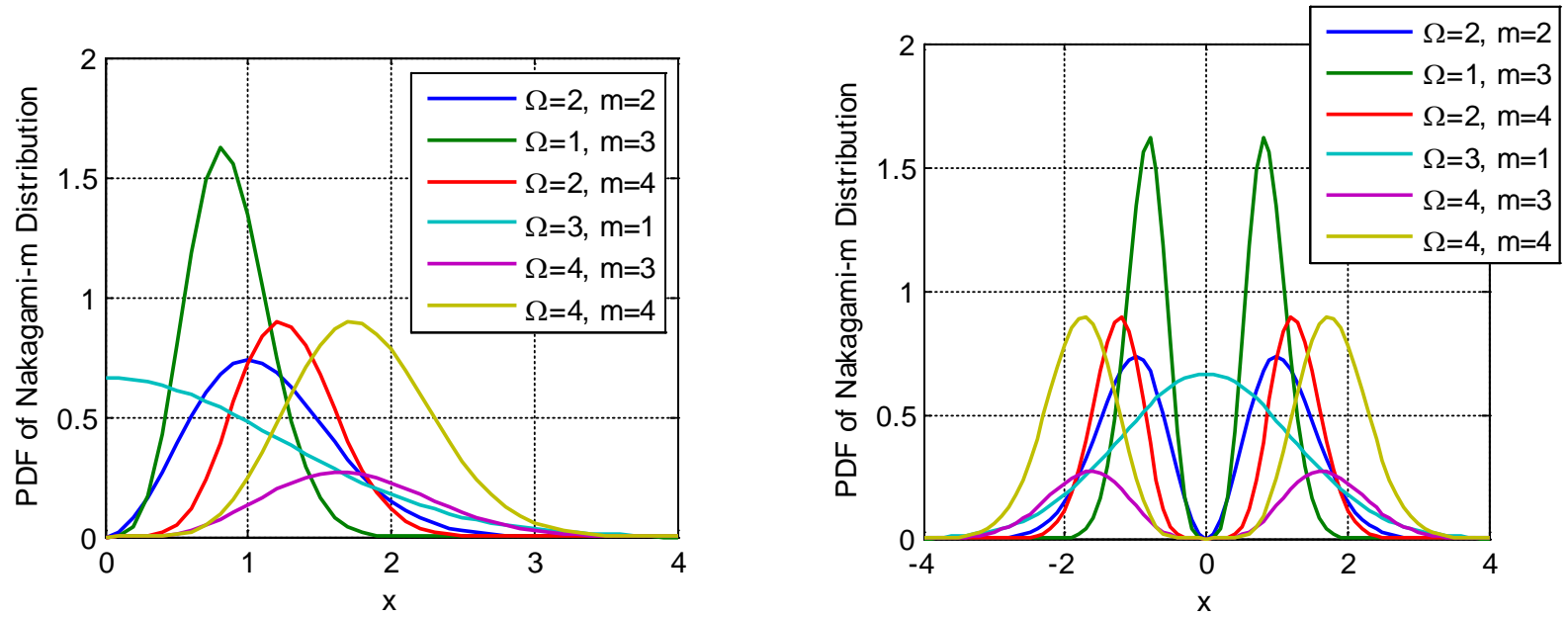

Fig. 2. One Side (a) and Double Side (b) PDF of Nakagami-m Distribution

Fig. 2(a). is the Nakagami-m PDF versus $x$, with $m$ and $\Omega$ taking different values from 1 to 4 , respectively. Fig. 1(b) shows that Nakagami-m is an even function, which symmetry to y axis. 


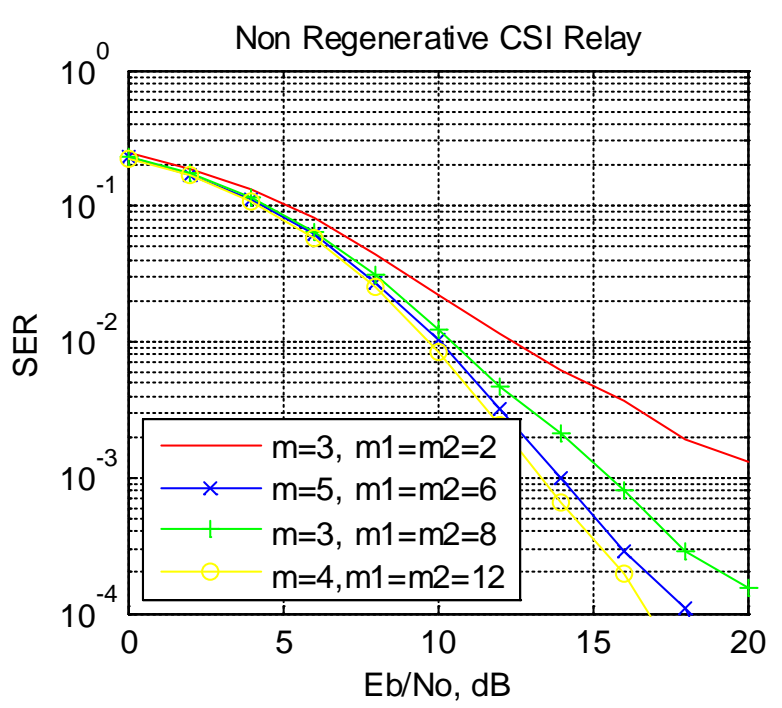

Fig. 3 SER of Non Regenerative CSI Relay

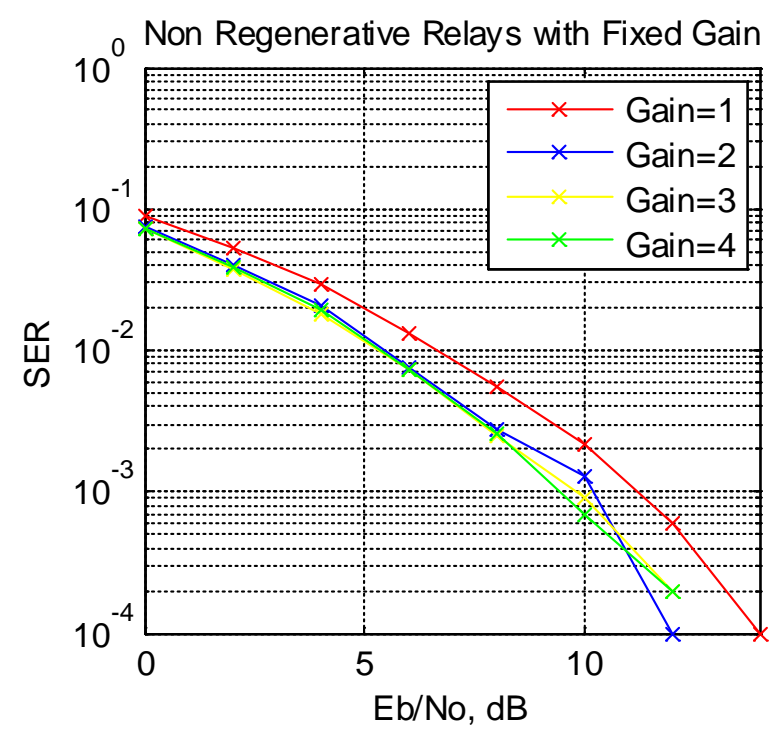

Fig. 5 SER of Fixed Gain Relay for BPSK

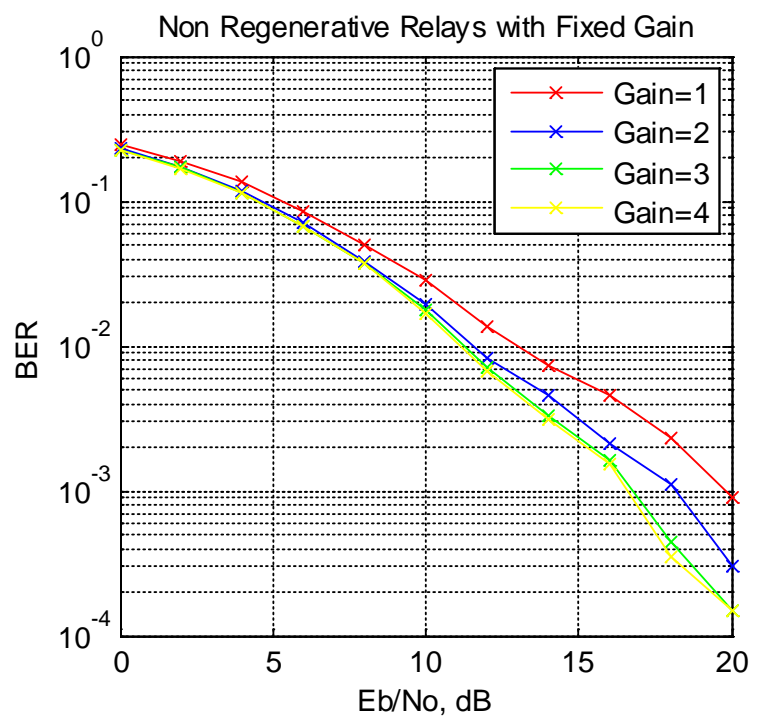

Fig. 7 BER of Fixed Gain Relay for QPSK

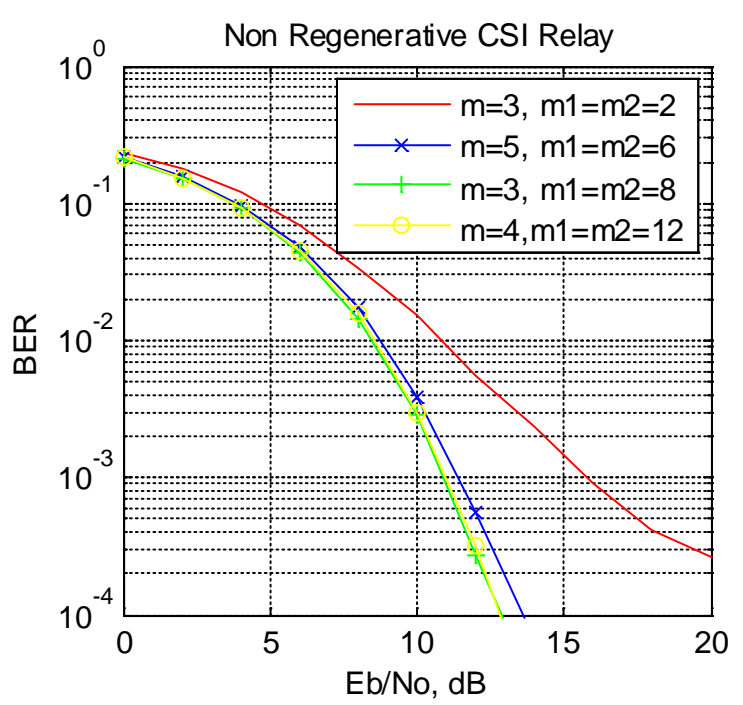

Fig. 4 BER of Non Regenerative CSI Relay

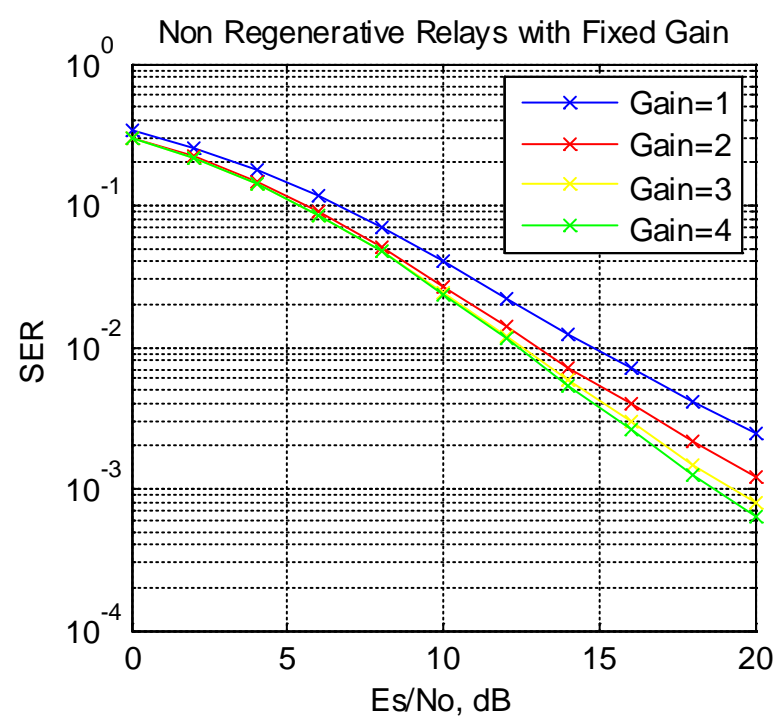

Fig.6 SER of Fixed Gain Relay for QPSK

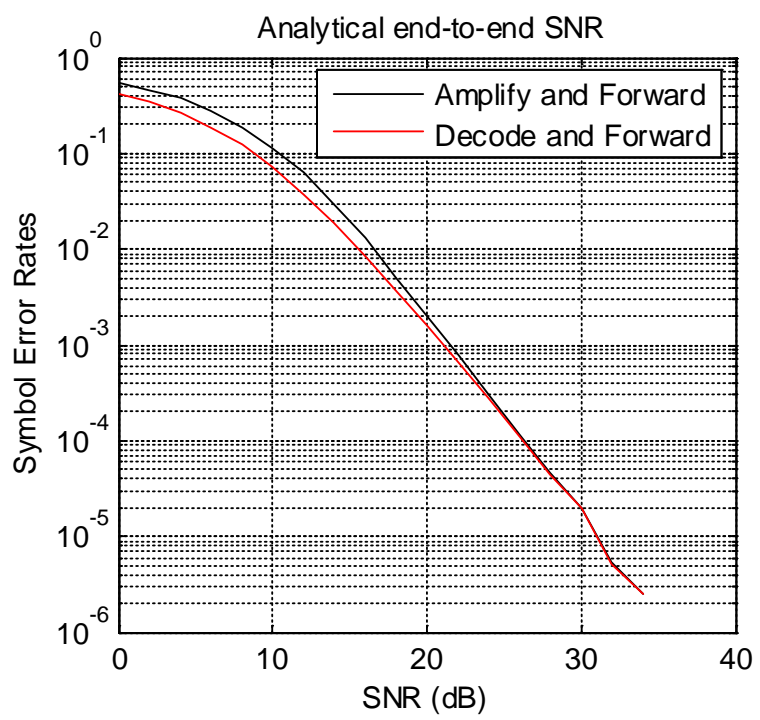

Fig. 8 QPSK comparison in Nakagami-m 
Fig. 3 is SER of non-regenerative channel state information relay in Nakagami-m fading channel; Fig. 4 is BER of non-regenerative channel state information relay in Nakagami-m fading channel; Fig. 5 is SER of non-regenerative with fixed gain relay using BPSK in Nakagami-m fading channel; Fig.6 is SER of non-regenerative with fixed gain relay using QPSK in Nakagami-m fading channel; Fig.7 is BER of non-regenerative with fixed gain relay using QPSK in Nakagami-m fading channel; Fig.8 is analytical end-to-end SNR vs. SER for QPSK comparison in Nakagami-m fading channel.

\section{Conclusion}

The Nakagami-m distribution has been widely used to describe the multipath fading statistics for its good fitting to experimental data. This work was devoted in the introduction, formulation and simulation Nakagami-m fading distribution. More precisely, we make a simulation study of the Nakagami-m distribution and investigate BER and SER of non-regenerative with fixed gain relay using BPSK, QPSK, respectively, in Nakagami-m fading channel. SER and BER of non-regenerative channel state information relay in Nakagami-m fading channel also investigated. Besides, the simulation study of wireless communication systems with non-regenerative fixed gain relays operating over Nakagami-m fading channels is also done. Moreover, the end-to-end signal-to-noise ratio (SNR) is simulated.

\section{Acknowledgement}

The author would like to thank the anonymous reviewers for their valuable comments that helped to improve the quality of this paper.

\section{References}

[1] V.Jagan Naveen, K.Raja Rajeswari, "Generation of Nakagami Fading Signals with Arbitrary Correlation and Fading Parameters”, International Journal of Future Generation Communication and Networking, Vol. 4, No. 2, June, 2011, pp. 49-68.

[2] Elina Pajala, Tero Isotalo, Abdelmonaem Lakhzouri, Elena Simona Lohan, “An improved simulation model for Nakagami-m fading channels for satellite positioning applications”, Proceedings of the $3^{\text {rd }}$ workshop on positioning, Navigation and Communication (WPNC'06), pp. 81-90.

[3] Dongya Shen, Yanni Cui, Aofeng Zhang, Yihuai Yang, Ke Wu, “A Simple Simulation Method for Nakagami Fading Channel”, 2010 International Conference on Microwave and Millimeter Wave Technology (ICMMT), pp. 160-163.

[4] M. Nakagami, “The m-Distribution: A General Formula of Intensity Distribution of Rapid Fading," Statistical Methods in Radio Wave Propagation, Pergamon Press: Oxford, U.K., 1960, pp. 3-36.

[5] YIhuai Yang, Lifang Wang, Mingxiu Shen, A Simulation Study of Nakagami-m Distribution, Advanced Materials Research.

[6] George K. Karagiannidis, Dimitris A. Zogas, Nikos C. Sagias, Theodoros A. Tsiftsis†, and P. Takis Mathiopoulos, Multihop Communications with Fixed-Gain Relays over Generalized Fading Channels, IEEE Communications Society, Globecom 2004, pp36-40.

[7] M. K. Simon and M.-S. Alouini, Digital Communication over Fading Channels. 1st ed., New York: Wiley, 2000. 